\title{
Preparation of barium titanate-bismuth magnesium titanate ceramics with high Curie temperature and their piezoelectric properties
}

\author{
Satoshi WADA, ${ }^{\dagger}$ Keisuke YAMATO, Petr PULPAN, Nobuhiro KUMADA, Bong-Yeon LEE, ${ }^{*}$ \\ Takashi IIJIMA, ${ }^{*}$ Chikako MORIYOSHI** and Yoshihiro KUROIWA** \\ Interdisciplinary Graduate School of Medical and Engineering, University of Yamanashi, 4-4-37 Takeda, Kofu 400-8510 \\ *National Institute of Advanced Industrial Science and Technology, AIST Tsukuba Central 5, Tsukuba, Ibaraki 305-8565 \\ ** Department of Physical Science, Hiroshima University, 1-3-1 Kagamiyama, Higashihiroshima, Hiroshima 739-8526
}

\begin{abstract}
Barium titanate $\left(\mathrm{BaTiO}_{3}, \mathrm{BT}\right)$-bismuth magnesium titanium oxide $\left(\mathrm{Bi}\left(\mathrm{Mg}_{0.5} \mathrm{Ti}_{0.5}\right) \mathrm{O}_{3}, \mathrm{BMT}\right)$ solid solution system ceramics were prepared using nanoparticles in atmosphere to enhance Curie temperature (Tc) of $\mathrm{BT},{ }^{\circ} 2^{\circ} \mathrm{C}$, to much higher temperature. Optimization of calcination and sintering conditions resulted in a formation of a perovskite single-phase, and their densities were always greater than $94 \%$. The synchrotron XRD measurement revealed that the all ions in the ceramics had almost homogeneous distribution. Temperature dependence of dielectric property revealed that the BT-BMT system ceramics was typical relaxor materials, and for the $0.5 \mathrm{BT}-0.5 \mathrm{BMT}$ ceramics, the dielectric maximum was clearly observed at $360^{\circ} \mathrm{C}$. Finally, their apparent piezoelectric constant $\left(d^{*}\right)$ were measured by electric-field dependence of strain at room temperature, and the $d^{*}$ value was measured at around $60 \mathrm{pm} / \mathrm{V}$ for the $0.4 \mathrm{BT}-0.6 \mathrm{BMT}$ ceramics.
\end{abstract}

๑2010 The Ceramic Society of Japan. All rights reserved.

Key-words : Barium titanate, Bismuth magnesium titanium oxide, High Tc, Piezoelectric property, Relaxor

[Received May 6, 2010; Accepted July 15, 2010]

\section{Introduction}

Recently, lead-free ferroelectrics have become highly attractive materials from the viewpoint of providing a solution to the environmental problems associated with conventional ferroelectrics such as $\mathrm{Pb}(\mathrm{Zr}, \mathrm{Ti}) \mathrm{O}_{3}$ (PZT) ceramics. ${ }^{1)}$ However, compared with PZT ceramics, ${ }^{2)}$ their ferroelectric related properties are very poor. Therefore, many researchers have attempted to improve the piezoelectric properties of lead-free ferroelectrics, and gradual improvements have been achieved. ${ }^{1)-4)}$ Recently, chemically modified potassium niobate $\left(\mathrm{KNbO}_{3}, \mathrm{KN}\right)$ and sodium niobate $\left(\mathrm{NaNbO}_{3}\right)$ solid solution ceramics have been reported as new lead-free piezoelectrics with a new morphotropic phase boundary (MPB), and piezoelectric properties similar to those of PZT ceramics. ${ }^{5), 6)}$ However, there are still no lead-free piezoelectric materials with much higher piezoelectricity than that of PZT ceramics.

As considering piezoelectric applications, especially actuator device application at off-resonance, there were two important parameters, i.e., piezoelectric constant $(d)$ and Curie temperature (Tc). For example, barium titanate $\left(\mathrm{BaTiO}_{3}, \mathrm{BT}\right)$ ceramics is a typical lead-free ferroelectric material, and its piezoelectric constant, $d_{33}$, was reported as around $191 \mathrm{pC} / \mathrm{N} .^{2)}$ Recently, for fine-grained BT ceramics, much higher $d_{33}$ values were reported, i.e., Takahashi et al. reported that the introduction of fine domains with a size of $50 \mathrm{~nm}$ into these ceramics resulted in a high $d_{33}$ of $350 \mathrm{pC} / \mathrm{N},{ }^{7)}$ while Karaki et al. reported that the introduction of fine domains into these ceramics resulted in a high $d_{33}$ of $460 \mathrm{pC} / \mathrm{N}^{8}{ }^{8)}$ Moreover, for the [110] grain-oriented BT ceramics, the higher $d_{33}$ of $788 \mathrm{pC} / \mathrm{N}$ was reported by Wada

\footnotetext{
Corresponding author: S. Wada; E-mail: swada@yamanashi.ac.jp
}

et al. ${ }^{9)}$ However, its Tc was only $132^{\circ} \mathrm{C}$, and this low Tc made its piezoelectric application limited into transducer in water. Therefore, it is important to increase Tc of BT ceramics over $300^{\circ} \mathrm{C}$.

It was well known that there were some perovskite materials with higher Tc such as $\mathrm{KN}$ with $\mathrm{Tc}$ of $435^{\circ} \mathrm{C}$ and bismuth ferrite $\left(\mathrm{BiFeO}_{3}, \mathrm{BF}\right)$ with $\mathrm{Tc}$ of $830^{\circ} \mathrm{C},{ }^{10)}$ and some researchers already studied solid solution system ceramics between $\mathrm{BT}$ and the above perovskite materials. ${ }^{11), 12)}$ Currently, new bismuth perovskite materials with higher Tc became highly attractive, such as bismuth chromium oxide $\left(\mathrm{BiCrO}_{3}\right)$ with $\mathrm{Tc}$ of $470^{\circ} \mathrm{C},{ }^{13)}$ bismuth manganese oxide $\left(\mathrm{BiMnO}_{3}\right)$ with $\mathrm{Tc}$ of $500^{\circ} \mathrm{C}^{14)}$ and bismuth cobalt oxide $\left(\mathrm{BiCoO}_{3}\right)$ with $\mathrm{Tc}$ over $1,000^{\circ} \mathrm{C} .{ }^{15)}$ The preparation of these bismuth perovskite materials required high pressure over $6 \mathrm{GPa}$ and high temperature over $1,000^{\circ} \mathrm{C}$, and their high leakage current made always poling treatment difficult or impossible.

Recently, some researchers focused into tetragonal bismuth zinc titanium oxide $\left(\mathrm{Bi}\left(\mathrm{Zn}_{0.5} \mathrm{Ti}_{0.5}\right) \mathrm{O}_{3}\right.$, BZT) with Tc over $700^{\circ} \mathrm{C} .^{16)}$ This is because that it is expected that BZT has relatively low leakage current owing to low leakage current elements such as $\mathrm{Ti}$ and $\mathrm{Zn}$ ions as compared to high leakage current elements such as $\mathrm{Co}, \mathrm{Fe}$, and $\mathrm{Cr}$ ions. However, to prepare BZT ceramics, high pressure over $6 \mathrm{GPa}$ and high temperature over $900^{\circ} \mathrm{C}$ were required. Recently, Huang and Cann prepared lead-free BT-BZT solid solution system ceramics in atmosphere, and reported that this system had a limited solid solution at 34 molar\% of BZT and maximum Tc was around $160^{\circ} \mathrm{C} .{ }^{17)}$ On the other hand, Yamato et al. prepared BT-BZT system ceramics using oxide nanoparticles as raw materials in atmosphere, and reported that the system had a limited solid solution at 50 molar\% of BZT and maximum Tc was around $230^{\circ} \mathrm{C}^{18)}$ Moreover, they also reported higher loss tangent over $10 \%$ at room temperature for $0.5 \mathrm{BT}-0.5 \mathrm{BZT}$ ceramics, and this 
high loss tangent still made poling treatment very difficult. For BT-BZT ceramics, it can be considered that an origin of high loss tangent may be Bi vacancy and valence-changeable $\mathrm{Zn}$ ions. Therefore, if $\mathrm{Zn}$ ion can be replaced by more valence-stable ions such as $\mathrm{Mg}$, new lead-free piezoelectric materials with low loss tangent may be achieved. Khalyavin et al. prepared single crystal of bismuth magnesium titanium oxide $\left(\left(\mathrm{Bi}\left(\mathrm{Mg}_{0.5} \mathrm{Ti}_{0.5}\right) \mathrm{O}_{3}, \mathrm{BMT}\right)\right)$ using high pressure of $6 \mathrm{GPa}$, and this crystal structure was assigned to orthorhombic (Pnnm). ${ }^{19)}$ This symmetry is exactly the same as that of antiferroelectric lead zirconate $\left(\mathrm{PbZrO}_{3}\right)$, which suggested that BMT may be also antiferroelectrics. However, Suchomel and Davis prepared lead titanate $\left(\mathrm{PbTiO}_{3}\right.$, PT) and BMT solid solution system ceramics in atmosphere, and reported that this system had MPB between tetragonal and rhombohedral. ${ }^{20)}$ Therefore, it is very important to investigate new lead-free BT-BMT solid solution system.

Therefore, in this study, the BT-BMT system ceramics with various chemical compositions were prepared using nanoparticles in atmosphere, and their piezoelectric properties were investigated.

\section{Experimental procedure}

The BT-BMT system ceramics were prepared by a conventional sintering method in atmosphere. As raw materials, $\mathrm{Bi}_{2} \mathrm{O}_{3}$ (Rare Metallic Co., LTD., >99.999\%), $\mathrm{TiO}_{2}$ (Ishihara Sangyo Kaisha LTD., $>99.9 \%$, size: $7 \mathrm{~nm}$ ), $\mathrm{MgO}$ (Ube Materials Industries Co., LTD., $>99.9 \%$, size: $20 \mathrm{~nm}$ ) and $\mathrm{BaCO}_{3}$ (Ube Materials Industries Co., LTD., >99.9\%, size: $40 \mathrm{~nm}$ ) were used. These powders were weighed at proper molar ratios of $(1-x)$ BT $-x$ BMT $(0<x<0.7)$, and then mixed by ball milling. The mixed powders were calcined at $950^{\circ} \mathrm{C}$ for $6 \mathrm{~h}$, and then crashed by ball milling with polyvinyl butyral (PVB, 2 wt \%) as a binder, and dried at $130^{\circ} \mathrm{C}$. The powders were meshed and then pressed into green pellets using a uniaxial press at room temperature. After the binder was burned out at $700^{\circ} \mathrm{C}$ for $10 \mathrm{~h}$, the pellets were sintered at various temperatures from 975 to $1290^{\circ} \mathrm{C}$ for $2 \mathrm{~h}$.

As in the first characterization, the absolute density was measured by Archimedes method, while the relative density was calculated using a theoretical density estimated from lattice parameters using a laboratory XRD (Rigaku RINT2000, $\mathrm{Cu} \mathrm{K} \alpha$, $50 \mathrm{kV}, 30 \mathrm{~mA}$ ). High-intensity synchrotron-radiation XRD data were collected at beam line BL02B2 of the synchrotron radiation facility, SPring- 8 . The diffraction patterns were analyzed by the Rietveld method using TOPAS software (Bruker axs, Version 2.1). For the electric measurements, the ceramics were polished and cut into sizes of $2 \times 2 \times 0.5 \mathrm{~mm}^{3}$. Silver electrodes were printed and fired on the top and bottom surfaces with an area of $2 \times 2 \mathrm{~mm}^{2}$. The dielectric properties were measured at various frequencies from 0.1 to $10 \mathrm{MHz}$ from 20 to $450^{\circ} \mathrm{C}$ using an impedance analyzer (Agilent, HP4294A). The strain vs. electricfield (S-E) curves were measured at room temperature and 0.1 and $1 \mathrm{~Hz}$ using a ferroelectric character evaluation system, and this slope of the S-E curve was regarded as an apparent piezoelectric constant $\left(d^{*}\right)$ value.

\section{Results and discussion}

\subsection{Preparation of BT-BMT system ceramics by conventional sintering method}

In this study, $(1-x) \mathrm{BT}-x \mathrm{BMT}$ system ceramics with various chemical compositions were prepared in atmosphere. First, for the BT-BMT system ceramics, the optimum sintering temperatures for each chemical composition were investigated. This is

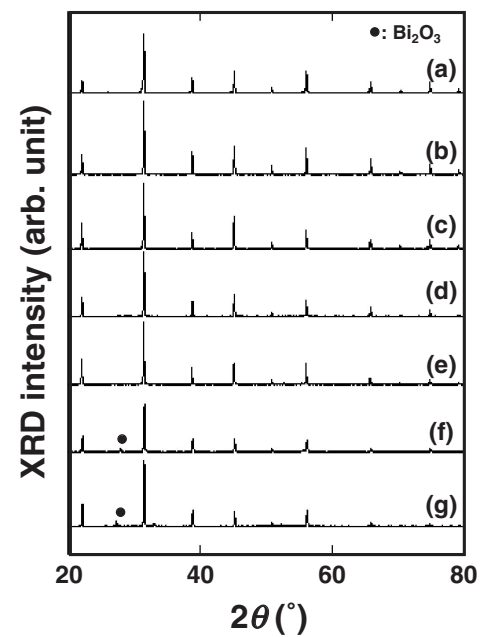

Fig. 1. XRD patterns measured at room temperature for the (a) 0.9BT$0.1 \mathrm{BMT}$, (b) $0.8 \mathrm{BT}-0.2 \mathrm{BMT}$, (c) 0.7BT-0.3BMT, (d) 0.6BT-0.4BMT, (e) $0.5 \mathrm{BT}-0.5 \mathrm{BMT}$, (f) $0.4 \mathrm{BT}-0.6 \mathrm{BMT}$, and (g) $0.3 \mathrm{BT}-0.7 \mathrm{BMT}$ ceramics.

because that it is expected that sintering temperature should decrease with increasing Bi contents. Thus, two typical chemical compositions such as BT and 0.5BT-0.5BMT were selected, and each optimum sintering temperature was determined as functions of density and dielectric loss tangent at $300 \mathrm{~Hz}$ and room temperature. By using the above-optimized temperatures, the sintering temperatures for ceramics with other compositions were determined assuming a linear relationship between sintering temperature and chemical composition. Sintered pellets were orange-colored, and their relative densities were always greater than $94 \%$. Figure 1 shows XRD measurement results for a series of the BT-BMT system ceramics. From Fig. 1, it was revealed that for all the BT-BMT ceramics, the perovskite phase was clearly observed, although in the BMT-rich ceramics with BMT contents over 60 molar\%, small amounts of $\mathrm{Bi}_{2} \mathrm{O}_{3}$ were observed as secondary phases. Therefore, it was confirmed that for the BTBMT ceramics, to prepare perovskite single phases by an atmosphere sintering, BMT contents must be limited below 60 molar\%. On the other hand, for BT-BZT system ceramics, to prepare perovskite single phases by an atmosphere sintering, BZT contents must be limited below 50 molar\%. This suggested that perovskite phase for BT-BMT is more stable as compared to that for BT-BZT, but, at present, we cannot explain this difference.

To confirm chemical homogeneity of the BT-BMT system ceramics, synchrotron XRD measurement was performed at high temperature of $475^{\circ} \mathrm{C}$. As the results, all of crystal structure for the BT-BMT system ceramics was assigned to cubic phase, and full width at half maximum (FWHM) of 222 peaks for the BTBMT system ceramics was much smaller than that for pure BT ceramics, which suggested that all of cations in these ceramics had almost homogeneous distribution. This is because that if there is inhomogeneous distribution for these cations in the ceramics, their FWHM must be much broader than that of pure BT. Next, synchrotron XRD measurement was performed at room temperature, and 222 peaks at room temperature were symmetric or very slight asymmetric shape, which suggested that this symmetry should be assigned to pseudo-cubic or rhombohedral. To confirm this hypothesis, Rietveld analysis was applied, and for $0.4 \mathrm{BT}-0.6 \mathrm{BMT}$, the crystal structure at room temperature 


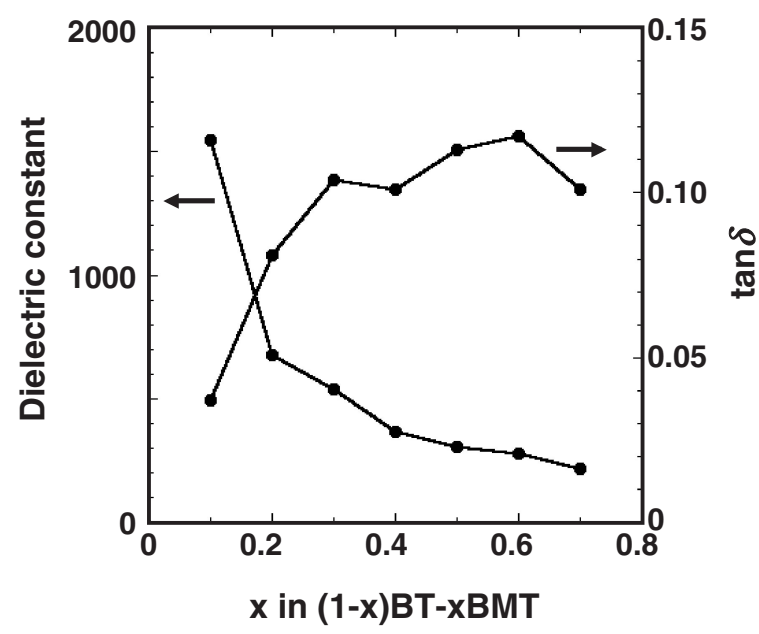

Fig. 2. The BMT content dependence of dielectric constant and loss tangent measured at $100 \mathrm{kHz}$ and room temperature for the BT-BMT system ceramics.

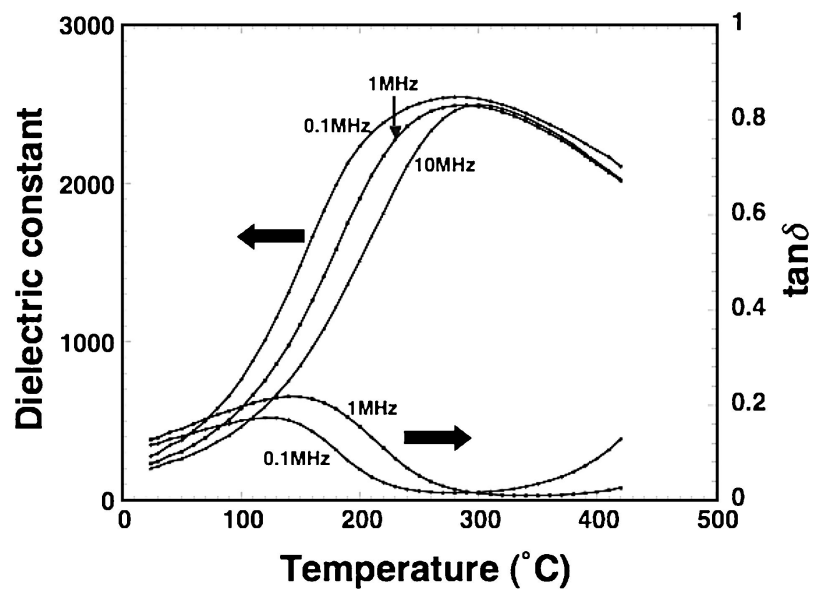

Fig. 3. Temperature dependence of the dielectric constant for the $0.4 \mathrm{BT}-0.6 \mathrm{BMT}$ ceramics.

was assigned to rhombohedral $R 3 \mathrm{~m}$ symmetry. However, to determine precise crystal structure, more investigation must be required.

\subsection{Dielectric properties of BT-BMT system ce- ramics}

First, dielectric properties for the BT-BMT system ceramics were measured at $100 \mathrm{kHz}$ and room temperature. Figure 2 shows BMT content dependence of dielectric constant and loss tangent. From Fig. 2, dielectric constant decreased from 1500 to 200 with increasing BMT contents, while loss tangent increased with increasing BMT contents and saturated at around $10 \%$. For BT-BZT system ceramics, loss tangent was almost $15 \%,{ }^{18)}$ and loss tangent for BT-BMT ceramic was slightly smaller than that for BT-BMT ceramics. By substitution of $\mathrm{Zn}$ site by $\mathrm{Mg}$ ions, it was expected to reduce loss tangent drastically, but the decrease of loss tangent was just by smaller than $5 \%$. These results suggested that for BT-BMT and BT-BZT system, higher loss tangent might be originated from $\mathrm{Bi}$ vacancy or other defect structure.

Next, to investigate Tc for the BT-BMT ceramics, the temperature dependence of the dielectric constant was investigated from 20 to $450^{\circ} \mathrm{C}$. As a typical example, Fig. 3 shows the

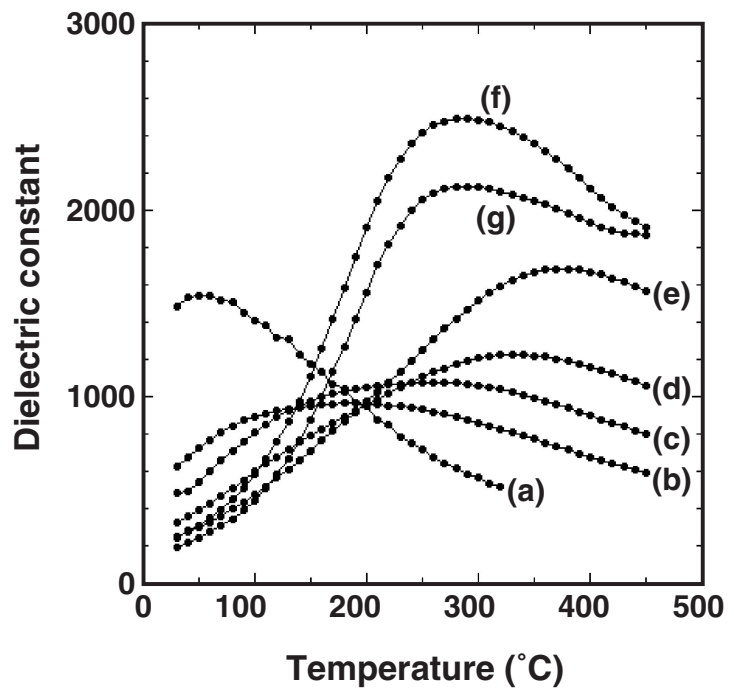

Fig. 4. Temperature dependence of the dielectric constant measured at $1 \mathrm{MHz}$ for the (a) $0.9 \mathrm{BT}-0.1 \mathrm{BMT}$, (b) $0.8 \mathrm{BT}-0.2 \mathrm{BMT}$, (c) $0.7 \mathrm{BT}-$ $0.3 \mathrm{BMT}$, (d) 0.6BT-0.4BMT, (e) 0.5BT-0.5BMT, (f) 0.4BT-0.6BMT, and $(\mathrm{g})$ 0.3BT-0.7BMT ceramics.

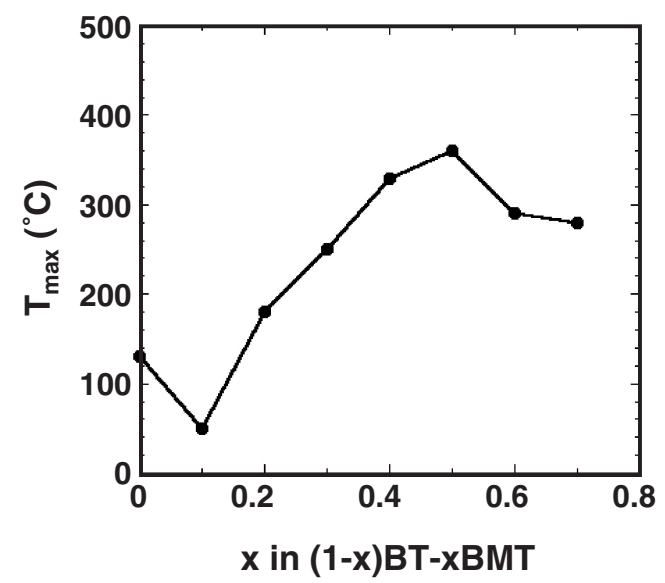

Fig. 5. The BMT content dependence of $\mathrm{T}_{\max }$ for the BT-BMT system ceramics.

temperature dependence of the dielectric constant for the 0.4BT$0.6 \mathrm{BMT}$ ceramics. From Fig. 3, it was clearly observed that the dielectric constant had strong frequency dependence, which revealed that BT-BMT system was typical relaxor system basis on the polar nanoregion. ${ }^{21)}$ This means that it is very difficult for relaxor system to determine Tc. Thus, through this manuscript, a dielectric maximum temperature measured at $1 \mathrm{MHz}\left(\mathrm{T}_{\max }\right)$ was regarded as corresponding value to Tc. Figure 4 shows temperature dependence of the dielectric constant measured at $1 \mathrm{MHz}$ for a series of BT-BMT ceramics. From Fig. 4, for the BT-BMT ceramics with BMT contents below 50 molar\%, the $\mathrm{T}_{\max }$ increased with increasing BMT contents, while for the BT-BMT ceramics with BMT contents over 50 molar $\%$, the $\mathrm{T}_{\max }$ decreased with increasing BMT contents, as shown in Fig. 5. At present, we cannot explain about this abnormal shift of $\mathrm{T}_{\max }$. For the BTBMT system, maximum $\mathrm{T}_{\max }$ was observed at $360^{\circ} \mathrm{C}$ for the $0.5 \mathrm{BT}-0.5 \mathrm{BMT}$ ceramics, which suggested that if this ceramics is poled, the poling state could be hold up to near $360^{\circ} \mathrm{C}$. Therefore, the BT-BMT system can become to one of the promising materials for high Tc piezoelectric applications. 


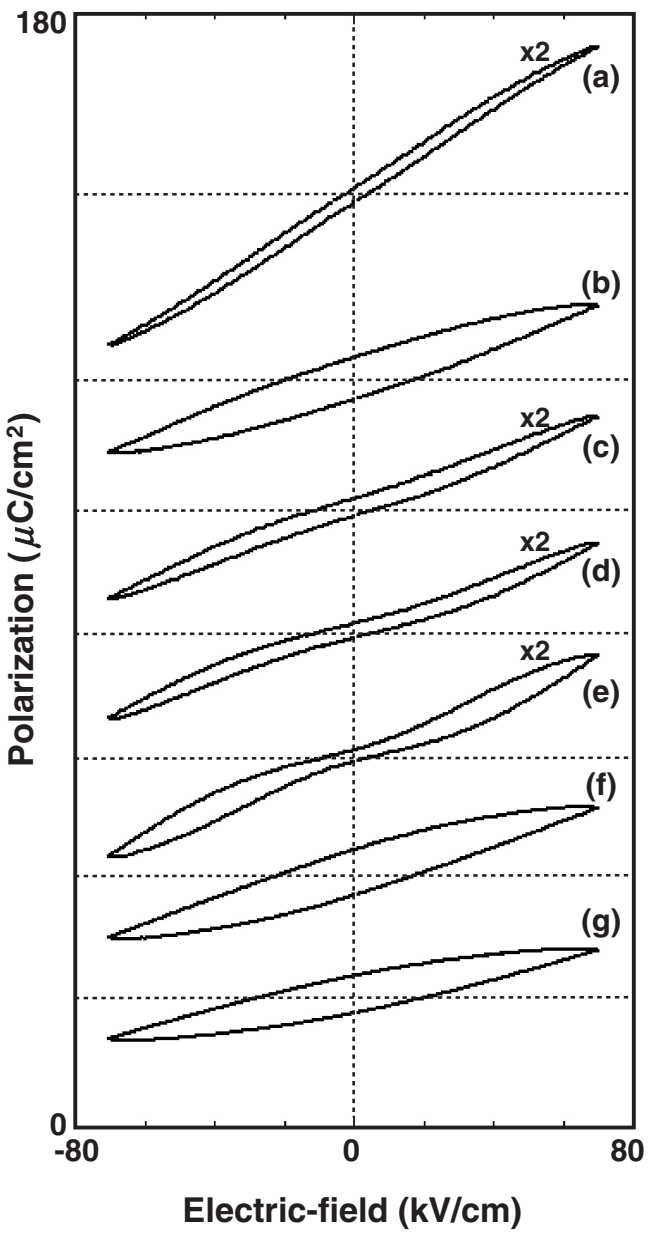

Fig. 6. P-E hysteresis loops measured at $1 \mathrm{~Hz}$ and room temperature for the (a) $0.9 \mathrm{BT}-0.1 \mathrm{BMT}$, (b) $0.8 \mathrm{BT}-0.2 \mathrm{BMT}$, (c) $0.7 \mathrm{BT}-0.3 \mathrm{BMT}$, (d) 0.6BT-0.4BMT, (e) 0.5BT-0.5BMT, (f) 0.4BT-0.6BMT, and (g) $0.3 \mathrm{BT}-0.7 \mathrm{BMT}$ ceramics: “ $\times 2$ ” in figure indicates that P-E loop was plotted using measurement values times 2 .

\subsection{Electric-field induced-strains of BT-BMT sys- tem ceramics}

Finally, as piezoelectric related properties, $\mathrm{P}-\mathrm{E}$ hysteresis loop and $\mathrm{S}-\mathrm{E}$ curves were measured at $1 \mathrm{~Hz}$ and room temperature for a series of the BT-BMT ceramics. Figure 6 shows P-E hysteresis loops while Fig. 7 shows S-E curves for the BT-BMT system ceramics. From Figs. 6 and 7, for the BT-BMT ceramics with BMT contents below 20 and over 60 molar\%, their P-E hysteresis loops and S-E curves were assigned to typical ferroelectric behaviors. On the other hand, for the BT-BMT ceramics with BMT contents from 30 to 50 molar\%, broad P-E double hysteresis loop were observed, which suggested that these ceramics might be assigned to antiferroelectric phase or ferroelectric phase with domain-pinning by certain defect structures. Moreover, S-E curves of these ceramics supported the above assignment. At present, it is very difficult to determine antiferroelectrics or ferroelectrics with domain pinning. From high loss tangent at dielectric measurement results, however, it was confirmed that there were high defect concentrations related to Bi vacancy or unknown defects, and the defect structure might be related to domain pinning sites. Thus, the unusual P-E double hysteresis loops, which observed for the BT-BMT ceramics with BMT contents from 30 to 50 molar\%, might be originated from defect related phenomena. To determine the origin of the $\mathrm{P}-\mathrm{E}$

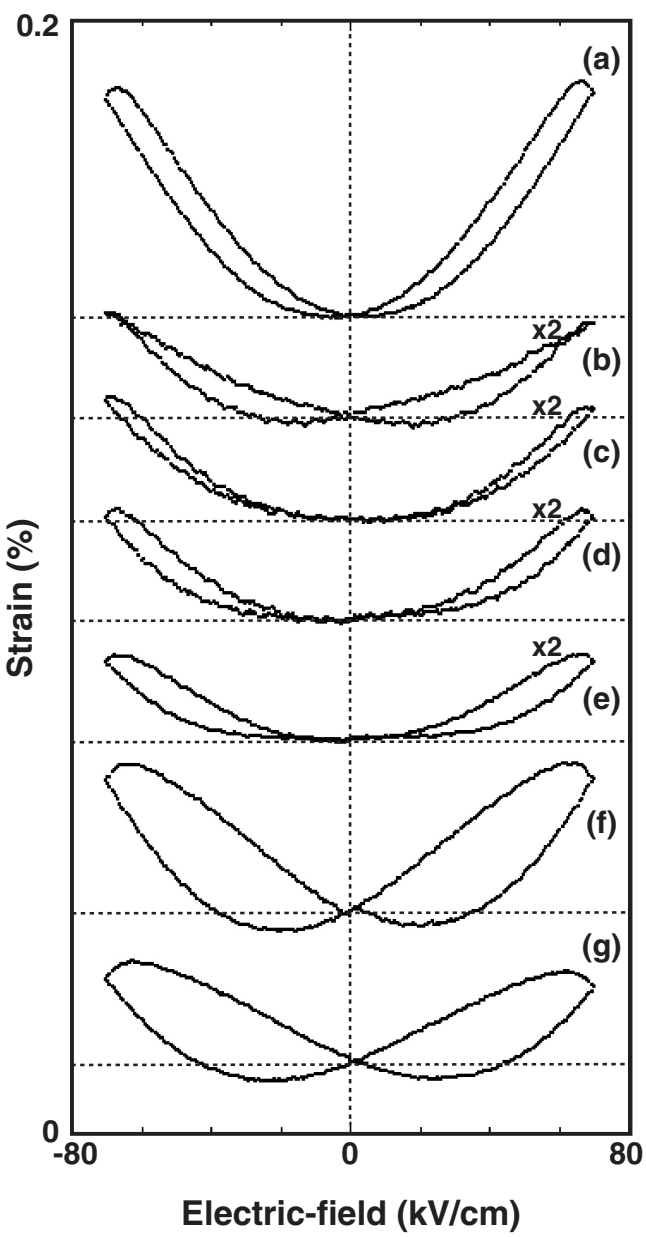

Fig. 7. S-E curves measured at $1 \mathrm{~Hz}$ and room temperature for the (a) 0.9BT-0.1BMT, (b) 0.8BT-0.2BMT, (c) 0.7BT-0.3BMT, (d) $0.6 \mathrm{BT}-$ $0.4 \mathrm{BMT}$, (e) $0.5 \mathrm{BT}-0.5 \mathrm{BMT}$, (f) $0.4 \mathrm{BT}-0.6 \mathrm{BMT}$, and (g) $0.3 \mathrm{BT}-$ $0.7 \mathrm{BMT}$ ceramics: " $\times 2$ " in figure indicates that $\mathrm{S}-\mathrm{E}$ curve was plotted using measurement values times 2 .

double hysteresis loops, much precise investigation will be required.

From Fig. 7, for the BT-BMT system ceramics with $\mathrm{T}_{\max }$ over $200^{\circ} \mathrm{C}$, maximum electric strain was obtained for the $0.4 \mathrm{BT}-$ $0.6 \mathrm{BMT}$ ceramics. Thus, precise P-E hysteresis loop and S-E curve of the $0.4 \mathrm{BT}-0.6 \mathrm{BMT}$ ceramics were measured at $0.1 \mathrm{~Hz}$ and room temperature. Figure 8 shows $\mathrm{P}-\mathrm{E}$ hysteresis loop and S-E curve for the 0.4BT-0.6BMT ceramics. From Fig. 8, residual polarization $\left(\mathrm{P}_{\mathrm{r}}\right)$ was estimated at around $3.5 \mu \mathrm{C} / \mathrm{cm}^{2}$ while the apparent piezoelectric constant $d^{*}$ value estimated from a slope of S-E curve at $80 \mathrm{kV} / \mathrm{cm}$ was around $60 \mathrm{pC} / \mathrm{N}$. It should be noted that this $d^{*}$ value of $60 \mathrm{pC} / \mathrm{N}$ was measured using the sample with high loss tangent of around $12 \%$ at $100 \mathrm{kHz}$. Thus, if lower dielectric loss tangent than $5 \%$ is achieved for the BT-BMT system ceramics, much larger electric strain may be obtained. At present, we are working to improve crystal quality by means of inhibition of vaporization of bismuth oxide.

\section{Conclusions}

In this study, the BT-BMT system ceramics were investigated as new high Tc lead-free piezoelectric materials. Optimization of calcination and sintering conditions resulted in a formation of a perovskite single-phase, and their densities were always greater 

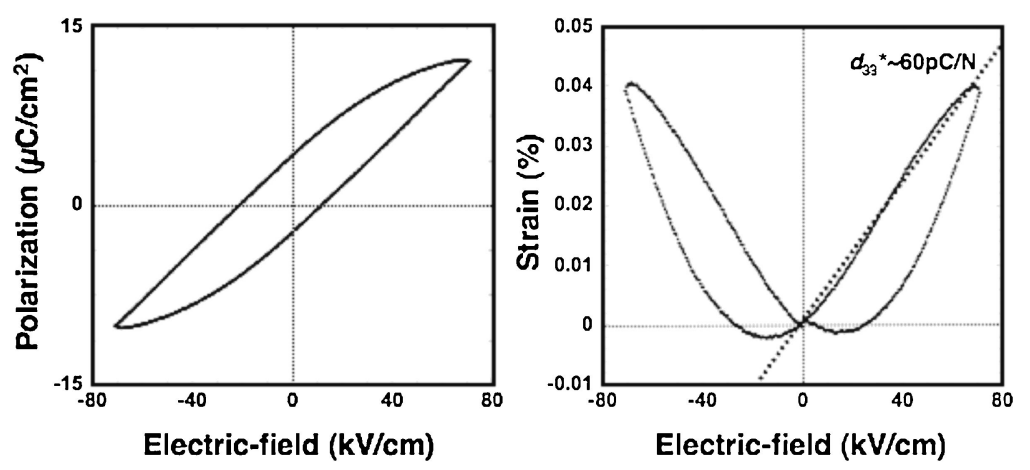

Fig. 8. P-E hysteresis loop and S-E curve measured at $0.1 \mathrm{~Hz}$ and room temperature for the $0.4 \mathrm{BT}-0.6 \mathrm{BMT}$ ceramics.

than $94 \%$. It was confirmed that the BT-BMT system ceramics exhibited high dielectric loss tangent of around $10 \%$. Moreover, temperature dependence of dielectric property revealed that for the $0.5 \mathrm{BT}-0.5 \mathrm{BMT}$ ceramics, the maximum $\mathrm{T}_{\max }$ of $360^{\circ} \mathrm{C}$ was clearly observed. Finally, their piezoelectric properties were measured using a slope of S-E curve at room temperature, and for the 0.4BT-0.6BMT ceramics, the apparent piezoelectric constant $d^{*}$ was measured at around $60 \mathrm{pC} / \mathrm{N}$. Thus, the BTBMT system can be one of the promising materials of high Tc piezoelectrics.

Acknowledgements We would like to thank Mr. F. Okada and Mr. S. Sano of Ube Material Industries Co., Ltd. for providing the high-purity $\mathrm{BaCO}_{3}$ and $\mathrm{MgO}$ nanoparticles. We would also like to thank Dr. K. Kato and Dr. M. Takata of SPring-8 for their help in XRD measurements. The experiment at SPring- 8 was carried out under Program No. 2008B1796. This study was partially supported by Grants-in-Aid for Scientific Research (16656201) from the Ministry of Education, Culture, Sports, Science and Technology, Japan (MEXT) and Elements Science and Technology Project from MEXT.

\section{References}

1) M. Demartin Maeder and D. Damjanovic, in "Piezoelectric Materials in Devices," ed. by N. Setter, N. Setter, Lausanne (2002) p. 389.

2) B. Jaffe, W. R. Cook, Jr. and H. Jaffe, "Piezoelectric Ceramics," Academic Press, New York (1971) p. 135.

3) F. Jona and G. Shirane, "Ferroelectric Crystals," Dover, New York (1993) p. 108.

4) Y. Zu, "Ferroelectric Materials and their Applications," NorthHolland, Amsterdam (1991) p. 101.

5) Y. Saito, H. Takao, T. Tani, T. Nonoyama, K. Takatori, T. Homma, T. Nagata and M. Nakamura, Nature, 432, 84-87 (2004).

6) Y. Guo, K. Kakimoto and H. Ohsato, Appl. Phys. Lett., 85,
4121-4123 (2004).

7) H. Takahashi, Y. Numamoto, J. Tani and S. Tsurekawa, Jpn. J. Appl. Phys., 45, 7405-7408 (2006).

8) T. Karaki, K. Yan, T. Miyamoto and M. Adachi, Jpn. J. Appl. Phys., 46, L97-L98 (2007).

9) S. Wada, K. Takeda, T. Muraishi, H. Kakemoto, T. Tsurumi and T. Kimura, Jpn. J. Appl. Phys., 46, 7039-7043 (2007).

10) T. Mitsui, I. Tatsuzaki and E. Nakamura, "An Introduction to the Physics of Ferroelectrics," Gordon \& Breach, New York (1976) p. 404.

11) S. Wada, M. Nitta, N. Kumada, D. Tanaka, M. Furukawa, S. Ohno, C. Moriyoshi and Y. Kuroiwa, Jpn. J. Appl. Phys., 47, 7678-7684 (2008).

12) M. M. Kumar, S. Srinivas and V. Suryanarayana, J. Appl. Phys., 87, 855-862 (2000).

13) S. Niitaka, M. Azuma, M. Takano, E. Nishibori, M. Takata and M. Sakata, Solid State Ionics, 172, 557-559 (2004).

14) A. A. Belik, S. Iikubo, T. Yokosawa, K. Kodama, N. Igawa, S. Shamoto, M. Azuma, M. Takano, K. Kimoto, Y. Matsui and E. Takayama-Muromachi, J. Am. Chem. Soc., 129, 971-977 (2007).

15) A. A. Belik, S. Iikubo, K. Kodama, N. Igawa, S. Shamoto, S. Niitaka, M. Azuma, Y. Shimakawa, M. Takano, F. Izumi and E. Takayama-Muromachi, Chem. Mater., 18, 798-803 (2006).

16) M. R. Suchomel, A. M. Fogg, M. Allix, H. Niu, J. B. Claridge and M. J. Rosseinsky, Chem. Mater., 18, 4987-4989 (2006).

17) C.-C. Huang and D. P. Cann, J. Appl. Phys., 104, 024117 (2008).

18) K. Yamato, N. Kumada, C. Moriyoshi, Y. Kuroiwa, B.-Y. Lee, T. Iijima and S. Wada, Key Eng. Mater. 421-422, 38-41 (2010).

19) D. D. Khalyavin, A. N. Salak, N. P. Vyshatko, A. B. Lopes, N. M. Olekhnovich, A. V. Pushkarev, I. I. Maroz and Y. V. Radyush, Chem. Mater., 18, 5104-5110 (2006).

20) M. R. Suchomel and P. K. Davies, Appl. Phys. Lett., 86, 262905 (2005).

21) L. E. Cross, Ferroelectrics, 151, 305-320 (1994). 